\title{
Accessible Shopping Systems for Blind and Visually Impaired Individuals: Design Requirements and the State of the Art
}

\author{
Vladimir Kulyukin and Aliasgar Kutiyanawala*
}

\author{
Computer Science Assistive Technology Laboratory, Department of Computer Science, Utah State University, USA
}

\begin{abstract}
Independent grocery shopping is one of the most functionally challenging tasks for visually impaired and blind individuals. Many assistive shopping systems have been developed to address the problem of blind grocery shopping. In this article, we identify several design requirements for assistive shopping systems and analyze existing approaches to see how well they meet them. Our objective is to shed some light on possible research and development directions for the accessible blind shopping community and to offer designers of accessible shopping solutions evaluation tools that can be used as initial points of comparison.
\end{abstract}

Keywords: Accessible shopping, assistive technology.

\section{INTRODUCTION}

What would it take for blind and visually impaired (VI) individuals to shop at modern supermarkets independently? Many sighted shoppers, unless they closely know someone who is blind or VI, may never ask themselves this question, let alone ponder how their sensory-motor system seamlessly handles the amazing complexity of the modern supermarket that stocks an average of 45,000 products and has a median store size of 4,529 square meters [1]. They successfully navigate to the right aisles, locate the shelves with the desired products, identify the products on the shelves, read their ingredients, and deal with relocated or discontinued products.

Unlike sighted individuals, many blind and VI people do not shop independently. They typically rely on friends, relatives, volunteers, and store employees. When these individuals are unavailable, VI shoppers have to reschedule or postpone shopping trips. When they go to the supermarkets by themselves, they experience delays, waiting for store employees to assist them. Some staffers are unfamiliar with the store layout, others become irritated with long searches and requests to read aloud product ingredients, and still others do not have adequate English skills to read the products' ingredients or answer basic questions about the supermarket layout. These difficulties cause blind and VI shoppers to abandon searching for desirable products, settle for distant substitutes, or, in the worst case, abandon independent shopping altogether [2].

PeaPod (www.peapod.com) and similar home delivery services provide grocery shopping alternatives. However, such services are not universally available and, when available, require shoppers to schedule and wait for deliveries, thereby reducing personal independence and

*Address correspondence to this author at the Computer Science Assistive Technology Laboratory, Department of Computer Science, Utah State University, USA; Tel: 435512 0225;

E-mails: aliasgar.k@aggiemail.usu.edu making spontaneous shopping difficult. Nor do such services offer VI individuals opportunities to explore stores on their own. To overcome these access barriers, accessible shopping solutions are needed that increase personal independence, enable spontaneous shopping, and do not require that supermarkets undergo extensive technological adjustments. Such systems will make VI individuals less dependent on external assistance and improve their quality of life. Toward that end, in this article we will formulate a set of design requirements for accessible shopping solutions for VI individuals and use them to analyze several existing systems.

We would like to emphasize that training independent evaluators and having them evaluate various accessible shopping solutions in the field is beyond the scope of the work presented in this article. Such an evaluation, besides requiring substantial budgetary commitments, does not seem feasible to us at the moment, because some systems exist only as research prototypes while others exist only on paper as patents. Nonetheless, we hope that our analysis sheds some light on possible research and development (R\&D) directions for the accessible blind shopping community.

\section{DESIGN REQUIREMENTS}

The design requirements proposed in this article are informed by two focus groups on accessibility barriers to independent blind supermarket shopping conducted by the authors at the Utah State University's Center for Persons with Disabilities (USU CPD). The first focus group consisted of five VI individuals from Logan, Utah. The age ranged from 16 to 47 . Two used white canes; three used guide dogs. We met with each individual separately to minimize peer pressure. Each interview lasted one hour.

The second focus group with different participants was conducted during a regular monthly meeting of the Logan Chapter of the National Federation of the Blind (NFB) hosted by the USU CPD. This group consisted of six individuals from Cache Valley, Utah, all of whom held parttime or full-time jobs, used public transportation, and walked 
independently around their neighborhoods. The age ranged from 19 to 51. Four were white cane users; two used guide dogs. This focus group was an open forum and lasted three hours.

Written transcripts of both focus groups were combined into one electronic master transcript. We used ergonomics for one (EFO) to analyze the master transcript. EFO is an occupational therapy framework proposed by McQuistion in 1993 [3] that we successfully used in our previous accessible blind shopping investigations (e.g., [4-6]). EFO is a method of fitting tasks to individuals with disabilities who must repeatedly accomplish them in specific environments. Interviews or field studies are used to identify task functions, i.e., things that the individual must do in a given environment, and to match task functions with the individual's abilities. Unmatched or partially matched task functions are referred to as performance gaps. Solutions, called accommodation systems, are subsequently designed to bridge the performance gaps. Once identified, the performance gaps become design requirements for accommodation systems.

We found the EFO analysis to be particularly suitable for working with VI individuals in supermarkets, because, due to the uneven distribution of the VI population [7], it is difficult to find groups of statistically significant sizes with the exact same level of blindness and physical ability. Variability in vision and physical ability, primarily due to age or onslaught of blindness, within the locally available group is the norm. Another advantage of the EFO analysis, although by no means unique, is that it establishes a meaningful framework for comparing different accommodation systems operating in the same environment.

Table 1. Task Functions

\begin{tabular}{|c|c|}
\hline Function Name & Function Description \\
\hline \hline TF1 & Shopping list preparation \\
\hline TF2 & Getting to supermarket \\
\hline TF3 & Finding products in store \\
\hline TF4 & Getting to cash registers \\
\hline TF5 & Paying \\
\hline TF6 & Getting to exit \\
\hline TF7 & Getting home \\
\hline
\end{tabular}

Our analysis of the master transcript resulted in the identification of seven task functions given in Table 1. We will not address TF2, TF5, and TF7 in this article. Task functions TF2 and TF7 pertain to outdoor blind navigation, a separate research area with a vast $R \& D$ literature. Although TF5 was identified as a task function by our focus groups, it did not introduce a performance gap, because all participants indicated that they handled payment with credit cards. We readily acknowledge, however, that for VI individuals who prefer to pay cash, payment is a performance gap in that individual bills must be reliably recognized.
When analyzing TF1, we found it necessary to distinguish among planned shopping (complete shopping list available in store), spontaneous or opportunistic shopping when no shopping list is available in store, and mixed shopping when the shopping list is found to be incomplete or must be modified due to some information received in the store, e.g. a sales promotion.

The shopping list preparation for planned shopping is not a performance gap, because, assuming that the shopper has access to a PC with a screen reader, it reduces to the problem of eyes-free item selection from large data sets on PCs, which appears to be adequately addressed in the literature (e.g., [8, 9]). However, in opportunistic and mixed shopping, VI shoppers are likely to use mobile devices on which eyesfree item selection from large data sets is presently difficult due to limited input options (e.g., [10]). Therefore, if the accommodation system is to operate on a mobile device (e.g., a smart phone), eyes-free product selection and browsing user interfaces (UIs) are a performance gap and, consequently, a design requirement.

We decomposed TF3 into three sub-functions: TF3.1) store navigation; TF3.2) product search; and TF3.3) product identification (making sure that the found product is the one that the shopper really wants). All three sub-functions introduce performance gaps for our focus groups. It is important to note that store exploration, sometimes referred to as product or store browsing, is implied by TF3.2 and TF3.3, but not vice versa. In other words, the shopper cannot search for and retrieve products without browsing, i.e. inspecting and identifying individual products. However, the ability to just browse does not necessarily result in successful product retrievals due to the sheer numbers of products in modern supermarkets. Task functions TF4 (getting to cash registers) and TF6 (getting to the exit) do not introduce new performance gaps, because both reduce to sub-function TF3.1.

Another design requirement that we discovered during our analysis of the master transcript and informal conversations with VI individuals is the utilization of existing devices that VI individuals already own and know how to operate. Adding new devices not only increases the cost of ownership but also has a negative ergonomic impact, because VI persons already handle numerous navigation tools (e.g., white canes and guide dogs, Braille note takers, personal GPS devices) and everyday wearable objects (e.g., purses, backpacks, and bags). In addition, one should not forget that some VI shoppers must also handle their children, who accompany them to the store.

There is yet another aspect of accessible blind shopping to which we would like to draw the reader's attention, because it is often overlooked in the literature, and that is the degree of environmental adjustment. Accessible shopping systems must operate not only in research labs but also in real supermarkets with established business processes (e.g., restocking, inventory control, cleaning, advertisements, etc.). An exclusive focus on technological means to bridge performance gaps, in which the computer science and engineering literature abounds due to its concentration on the end user, runs the risk of developing accommodation systems that require unreasonable adjustments by the supermarket. 
We became aware of this in the course of our negotiations with the management of Lee's Market Place in Logan, Utah to allow us to use their store as a research site. One of the very first questions that we were asked was what hardware must be installed in the store, who would maintain it, and whether it would be disruptive to the shopper traffic. Subsequent trial runs and longitudinal experiments with VI shoppers turned our awareness into a firm conviction. One insight, slightly paraphrased, from the ecological systems theory [11] is immediately applicable: if the introduction of an accommodation system causes significant disruptions to the existing business processes, the supermarkets will resist or reject its adoption. Consequently, another design requirement is minimal environmental adjustment, with no environmental adjustment being the ideal. The designed requirements that we have identified are summarized in Table 2 .

Table 2. Design Requirements

\begin{tabular}{|c|c|}
\hline Requirement Name & Requirement Description \\
\hline \hline DR1 & Mobile product selection \\
\hline DR2 & Store navigation \\
\hline DR3 & Product search \\
\hline DR4 & Product identification \\
\hline DR5 & Utilization of existing devices \\
\hline DR6 & Minimal environ. adjustment \\
\hline
\end{tabular}

\section{ANALYSIS OF ACCESSIBLE SHOPPING SOLUTIONS}

Now that we have formulated several design requirements for accessible blind shopping systems, let us use them to analyze several existing approaches to see how well they meet these requirements. Our objective is to shed some light on possible R\&D directions for the accessible blind shopping community and to offer designers of accessible shopping solutions some evaluation tools that can be used as initial points of comparison.

Our analysis will use the following conventions. We will say that a system $\mathrm{S}$ meets a design requirement $\mathrm{R}$ when $\mathrm{S}$ has a software or hardware module specifically designed to meet $\mathrm{R}$. When the literature offers evidence that the designers of $\mathrm{S}$ are aware of $\mathrm{R}$ but have not had the resources to address it, we will say that $\mathrm{S}$ partially meets $\mathrm{R}$. When the literature offers no such evidence, we will say that $\mathrm{S}$ does not meet $\mathrm{R}$. In some cases, it is simply impossible to tell whether $\mathrm{S}$ meets, partially meets, or does not meet R. For example, if $\mathrm{S}$ is proposed in a patent but is not actually implemented. Moreover, the text of the patent or a description found in the literature makes no software or hardware commitments on how the system can be realized. In such cases, we will say that it is unknown if S meets R.

\subsection{RoboCart}

RoboCart was our first accessible blind shopping project that started in early 2004 [12] at the Computer Science Assistive Technology Laboratory of Utah State University

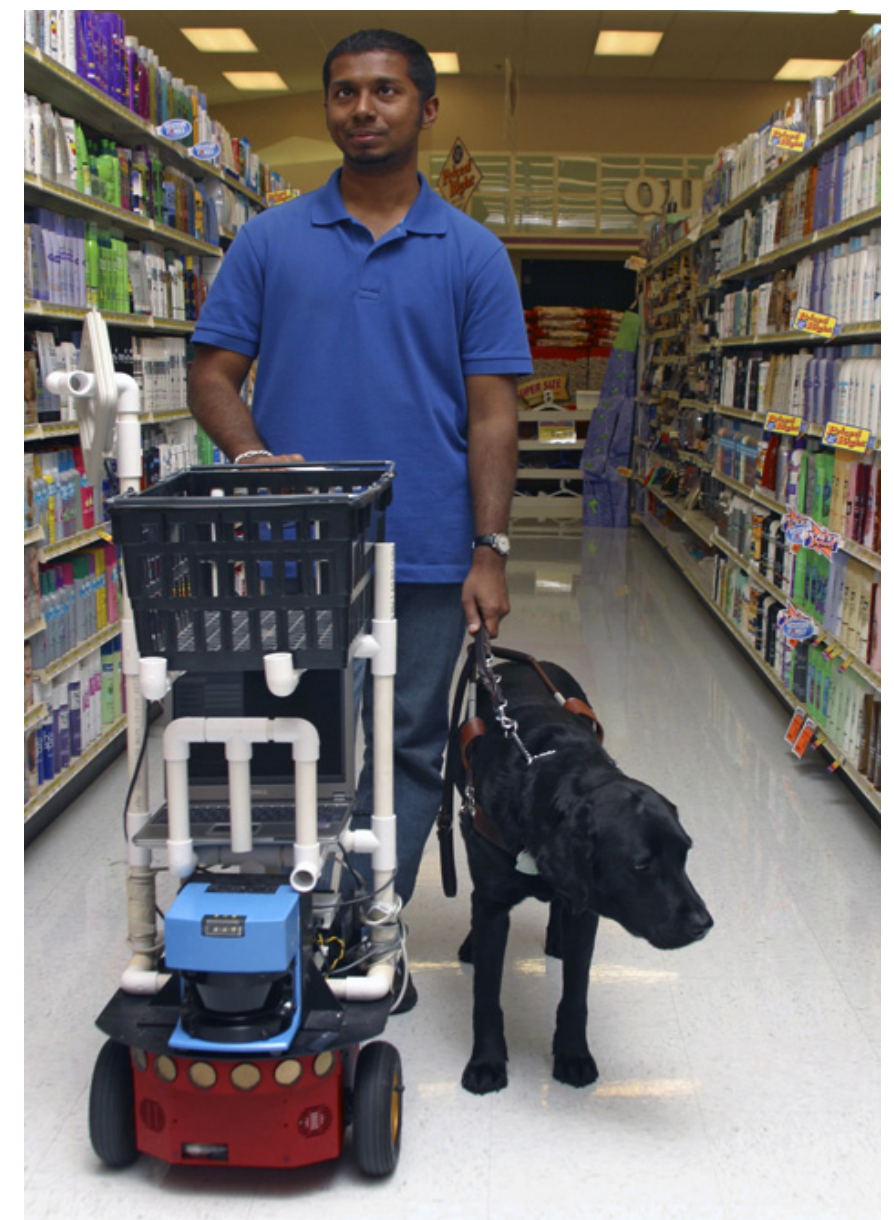

Fig. (1). RoboCart.

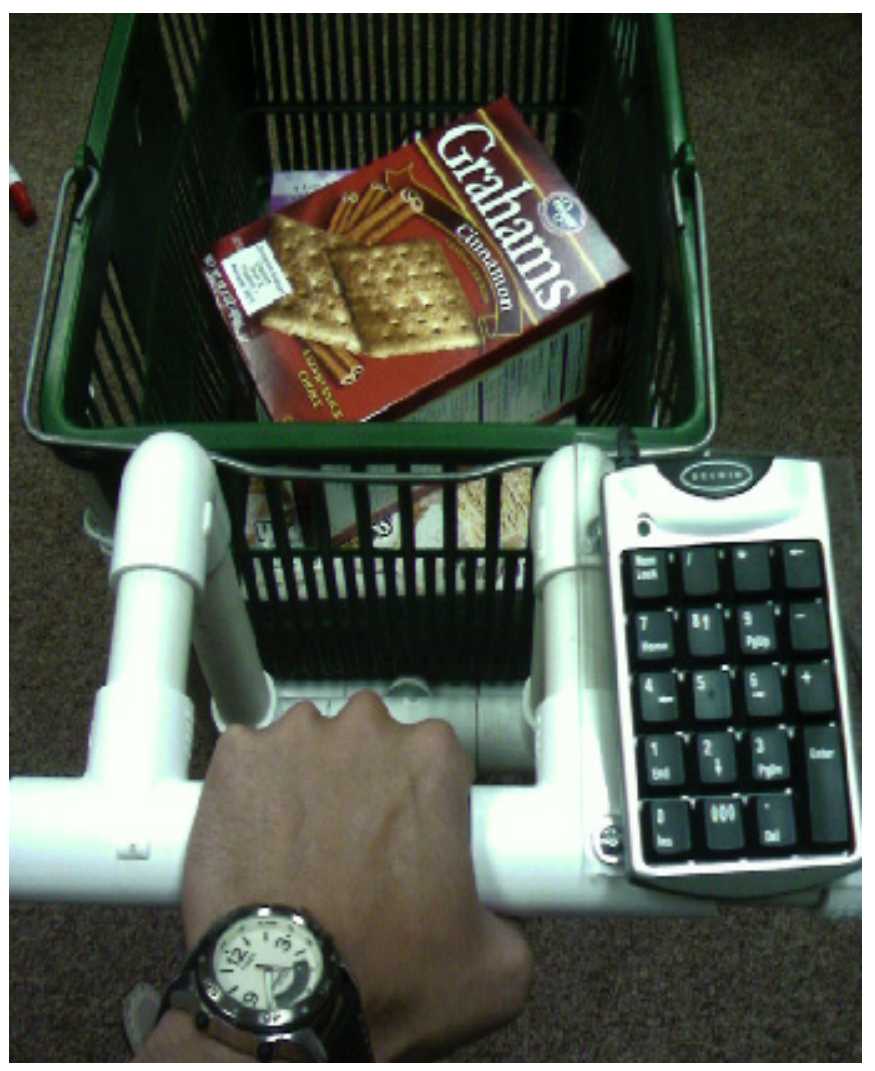

Fig. (2). Belkin keypad on handle. 
(USU CSATL). The objective was to design a robotic supermarket shopping assistant for blind and VI shoppers. A long-term collaborative agreement was negotiated with Lee's Market Place to grant us access to its supermarket in Logan, Utah for experimental purposes.

In 2004 - 2005, RoboCart was built on top of a commercial Pioneer 2DX platform (www.activmedia.com) to which a polyvinyl chloride (PVC) pipe structure was securely attached. The payload included a laptop, a SICK laser range finder, a Radio-Frequency Identification (RFID) reader, a $200 \mathrm{~mm} \times 200 \mathrm{~mm}$ RFID antenna, and a shopping basket (See Fig. 1).

For localization, RoboCart relied on passive RFID tags that we temporarily deployed at various locations in the store. Specifically, the tags were placed at the beginning and end of every aisle and at three different locations within each aisle. These tags allowed the robot to keep track of its position in each aisle and to correct global Markov localization errors. Several laser range finding techniques were developed for obstacle avoidance [13]. Single subject experiments were successfully executed with two VI participants. The participants' feedback allowed us to make iterative software and hardware changes to adjust the robot's navigation routines, i.e., improve its speed and make smoother turns.

In 2006, a small 10-key Belkin keypad was attached to the handle for product selection by list browsing and for finer robot control (See Fig. 2). The keypad was deliberately programmed to emulate the keypad of a cell phone. We also added a handheld wireless IT4600 SR barcode scanner.

After these hardware modifications were integrated, another two-month single subject study was executed in which each VI participant was asked to: 1) find RoboCart in the cart area, 2) use the keypad to select three grocery items, 3) navigate to shelf sections, 4) retrieve the selected items from the shelves by scanning shelf barcodes and reaching over them to retrieve the items, 5) place the items into RoboCart's basket, 6) navigate to a cash register, 7) place the items on the conveyer belt, 8) pretend to pay with a credit card, 9) navigate to the exit, 10) remove the shopping bags from RoboCart, and 11) leave the store. The database consisted of 24 products from aisles 9 and 10 in the supermarket. Seven trials were executed on three separate days for three different sets of products. The participant successfully completed all trials and retrieved all products [14].

In 2007, we focused on cognitive aspects of accessible blind shopping by developing a trichotomous spatial ontology of locomotor, haptic, and target spaces. The introduction of the target space (a shopper-centric subspace of the locomotor space where the shopper perceives that target product to be) extended the traditional locomotor vs haptic dichotomy in the blind navigation literature [15].

A longitudinal formal study was executed with ten VI participants recruited through the Utah National Federation of the Blind (NFB) Chapter. The supermarket experiments lasted four months with each participant having to execute fifteen runs on two different days. The 2006 product database was used. All trial runs were successfully completed by the participants and all research hypotheses verified.

As an accessible shopping accommodation system, RoboCart partially meets DR1 (mobile product selection), because its keypad with which the user selects products is programmed as a mobile phone keypad, which allows the shopper to select items by list browsing. The system meets DR2 (store navigation) through robot navigation and partially meets DR3 (product search) and DR4 (product identification) though a wireless barcode scanner. RobotCart addresses neither DR5 (utilization of existing devices) nor DR6 (minimal environmental adjustments).

\subsection{ShopTalk}

In early 2006, in parallel with our R\&D activities with RoboCart, we began our work on ShopTalk, a wearable system for independent blind supermarket shopping. We carefully considered several mobile phone platforms but found them unable to support the required computational loads without server support. As we had by then become fully aware of DR6 (minimal environmental adjustment), we wanted all computation to run on the system with no external server support. After considering several mobile phone platforms, we ruled them out as insufficiently powerful. Eventually we settled on the OQO 01 wearable computer, which we viewed as a temporary compromise solution with sufficient computational power and reasonable ergonomics.

The final ShopTalk hardware (See Fig. 3) consisted of the OQO computer, a Belkin numeric keypad, a Hand Held Products IT4600 SR wireless barcode scanner (See Fig. 4) and its base station, and a USB hub to connect all components. We used the ergonomic lessons we had learned from our field experiments with RoboCart and added two plastic stabilizers to the barcode scanner. The shopper could now place the stabilizers on shelf lips to align the scanner with shelf barcodes.

The equipment was placed in a small CamelBak backpack. The numeric keypad was attached by a Velcro strip to either left or right shoulder strap, depending on whether the shopper was left- or right-handed. Since all instructions were speech-based, the shopper was required to wear a small headphone.

ShopTalk was based on the following conceptual formula: independent blind shopping $=$ verbal route instructions + shelf barcode scans [16]. The system was our first attempt (and, to the best of our knowledge, the first attempt reported in the accessible shopping literature) to use MSI (Modified Plessey) shelf barcodes (See Fig. 4) as topological cues for product search and store navigation. MSI is a barcode symbology developed by the MSI Data Corporation (http://en.wikipedia.org/wiki/MSI_Barcode) used primarily for inventory control.

A key data structure in ShopTalk is the barcode connectivity matrix (BCM). The BCM associates MSI barcodes with aisles, aisle sides, shelf sections (groups of shelves), specific shelves in the shelf section, and relative positions on shelves. The BCM is used to generate the store navigation and product search and retrieval instructions. 
In 2007 - 2008, after two single subject studies at Lee's Market Place and one single subject study at Sweet Peas, a smaller independent natural foods store in Logan, Utah, ten VI participants were recruited for a formal longitudinal study. The product database included 4,297 products. The experiments, performed during regular business hours, had each participant shop for the same set of three randomly chosen products five times. The product retrieval rate was $100 \%$. All ten participants found all three products in every run [2].

A key finding of ShopTalk is that independent VI travelers can execute verbal template-based route and product search instructions in supermarkets with $100 \%$ accuracy. In effect, our experiments with the system have demonstrated that, for our sample of participants, verbal route directions were self-sufficient for successful blind navigation in a real supermarket.

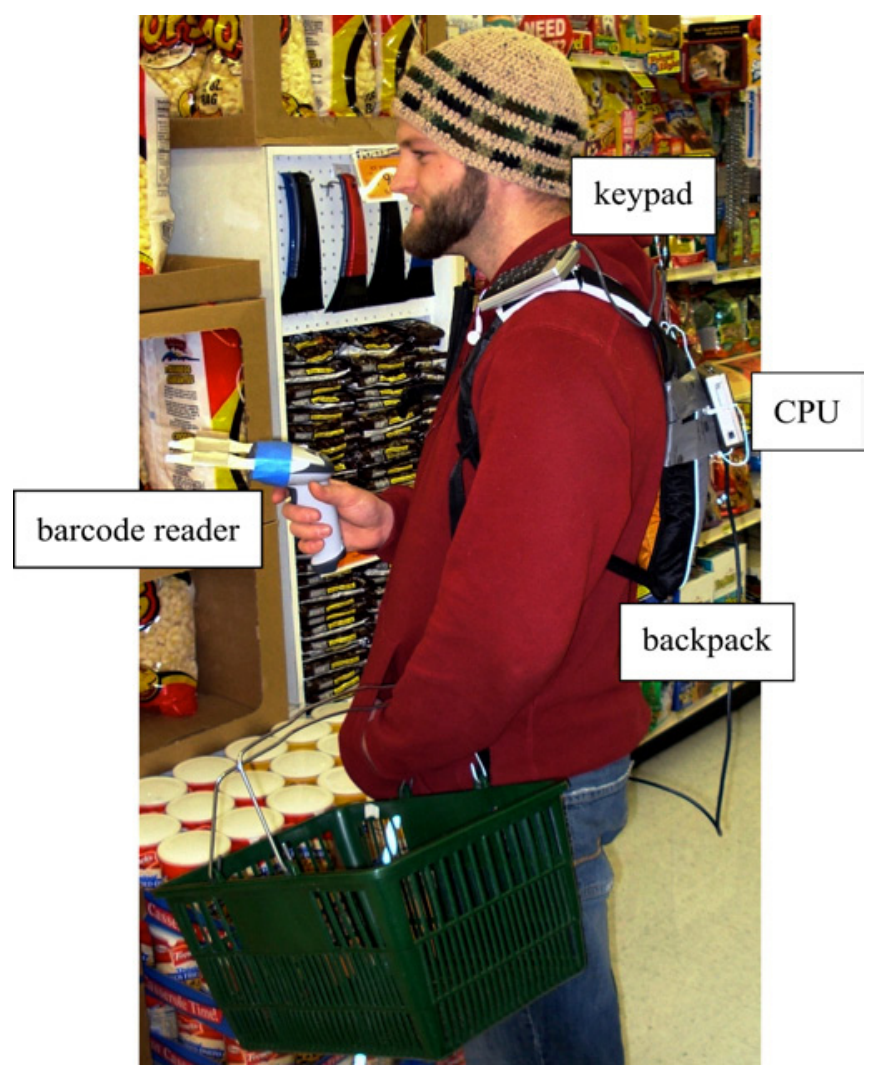

Fig. (3). ShopTalk.

As an accommodation system, ShopTalk does not meet DR1 (mobile product selection) in that a complete product list is assumed to be prepared and stored on the computational device. The system meets DR2 (store navigation) and DR3 (product search) by using shelf barcodes as topological cues during store navigation and product search. The system partially meet DR4 (product identification), because the user can verify the identity of the retrieved product by scanning its UPC barcode on the package. However, as we observed during the experiments, this scanner was difficult to use on softer packages (e.g., potato chips). ShopTalk does not meet DR5 (utilization of existing devices), because none of the hardware components used in the system are widely used by VI individuals. The system partially meets DR6 (minimal environmental adjustment). Although the supermarket is not required to install and maintain any new hardware, the system assumes access to the supermarket's inventory control system without which the BCM cannot be computed. Some supermarkets may not be willing to provide such access.

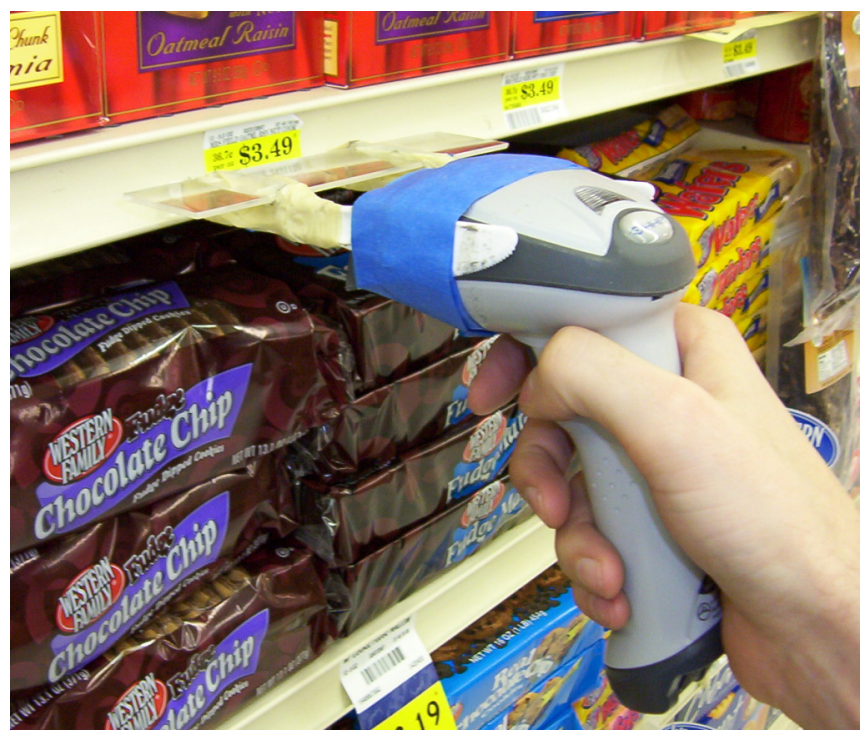

Fig. (4). MSI barcode on shelf scanned.

\subsection{GroZi}

GroZi [17] is an accessible shopping project at UC San Diego. The project has three components: 1) an accessible web site for blind and VI users to create grocery shopping lists in the comfort of their homes; 2) computer vision software for recognizing products and signs in stores; and 3) portable devices that can execute computer vision algorithms and give the user haptic and verbal feedback.

Figs. $(\mathbf{5}, \mathbf{6})$ (reproduced from http:/grozi.calit2.net/ with permission) show two such devices: the MoZi Box and the GroZi hand glove with a small portable camera and vibrating motors. In a typical GroZi use case, a VI shopper uses the web site to compile a shopping list of products and uploads it on the portable device. In the store, the shopper takes out the device and uses it to receive directions for each item in the shopping list. For example, if the shopper wears the glove and points at an aisle, the portable unit will indicate the components of that aisle and guide the shopper to the desired item.

The primary focus of the project has so far been on various object recognition algorithms for detecting items on the shopping list in continuous video streams [18]. The GroZi team's long-term objective is to enable the user to sweep the portable camera's field of view across the grocery shelves. When an item on the user's shopping list is detected in the video stream, the system will give the user either haptic or verbal guidance as to how to get to that item. In GroZi, there are two classes of images: in vitro and in situ. The in vitro images are images of products taken under ideal lighting and perspective conditions. The in situ images are obtained from actual video streams in the store. The former are used for training; the latter - for testing. 


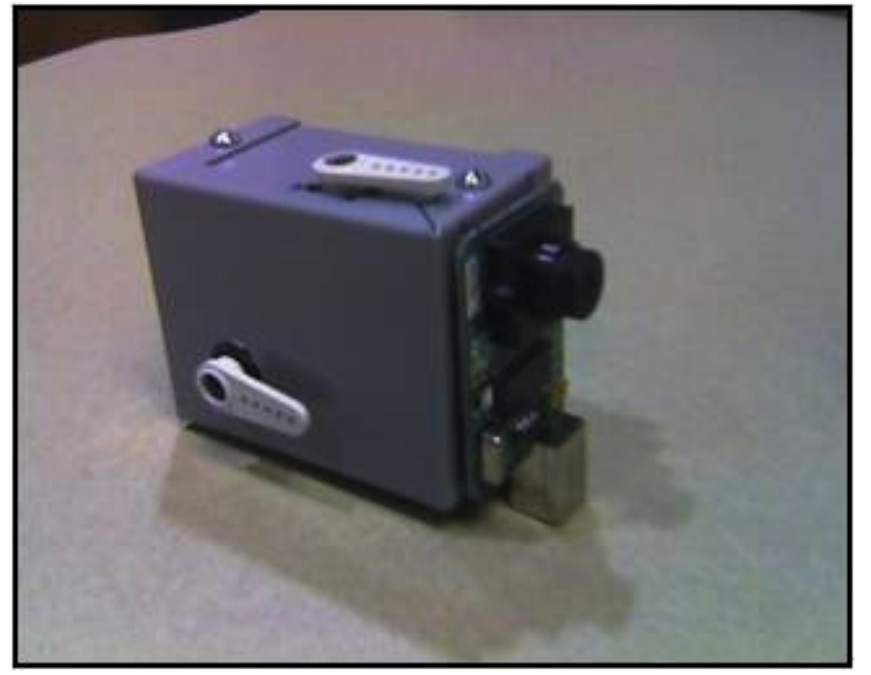

Fig. (5). MoZi box.

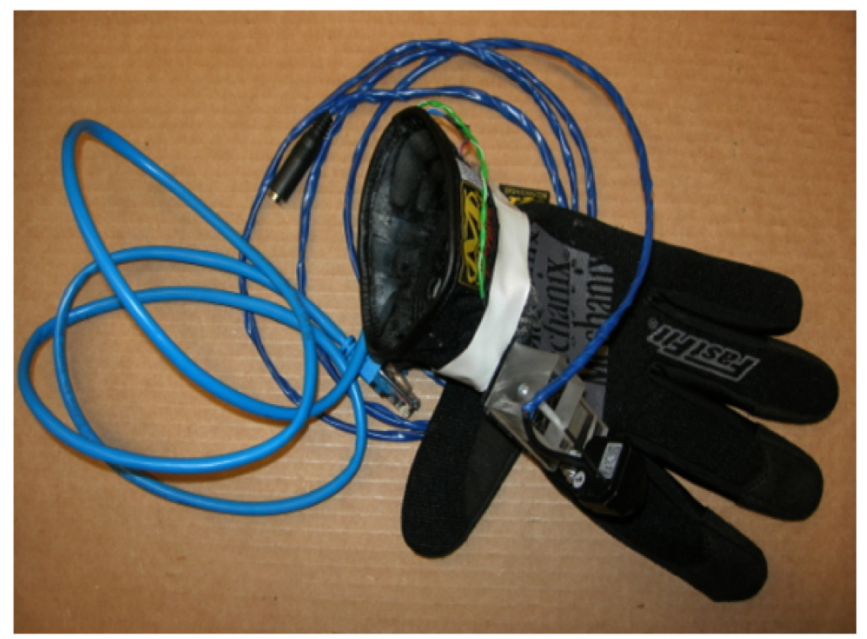

Fig. (6). GroZi glove.

As an accessible shopping accommodation system, GroZi does not meet DR1 (mobile product selection). As in ShopTalk, access to the complete shopping list is taken for granted, and the shopper is not supposed to modify the shopping list in the store. The system partially meets DR2 (store navigation). Although the current assumption is that the shopper is capable of navigating to each item's location independently, the designers of the system are aware that store navigation is a design requirement and plan to address it in their future work.

The system partially meets DR3 (product search). Once the shopper is in the right aisle, the system uses five haptic instructions in the two-dimensional plane of the shelf under exploration: up, down, left, right, step back - camera blurry. Theoretically speaking, these instructions should be sufficient to enable the VI shopper to find and retrieve a product from the shelf. However, this conjecture needs empirical verification in a real supermarket during regular operating hours.

The system partially meets DR4 (product identification) through various computer vision product identification algorithms. The developed algorithms (e.g., ShelfScanner [18]) can currently process about two frames per second. However, this speed comes at an ergonomic price: the shopper must wear a powerful laptop on his or her back. In principle, different procedures used in the algorithms can be parallelized. If such parallelization is achieved, it may be possible for the system to operate in real time without incurring significant performance costs.

The system does not meet DR5 (utilization of existing devices). Although each individual component of the GroZi hardware is completely-off-the-shelf, which makes for easier maintenance of the end deliverables, the end deliverables will still have to be assembled by hardware professionals and purchased by VI individuals in addition to the devices that they already own and know how to operate.

Whether the GroZi system meets DR6 (minimal environmental adjustment) is presently unknown. It depends on who will be ultimately responsible for maintaining the databases of the in vitro images required for training and where the servers hosting these databases will reside. If it is the supermarket that is required to maintain both the servers and the databases, then the system is unlikely to meet DR6 due to significant maintenance costs. The system could meet DR6 by outsourcing the maintenance of these resources to third parties, such as volunteer groups, local NFB chapters, etc. Of course, it remains to be seen how realistic and cost effective such outsourcing will be.

\section{4. iCare}

iCARE [19] is an accessible shopping project at Arizona State University. The project's objective is to design an ambient interactive shopping environment for VI shoppers. The ambient environment is assumed to provide reliable indoor navigation, an understanding of locations and contents of different sections and a user interface for querying product databases. The system design includes a PDA with Bluetooth, Wi-Fi, a screen reader, and an RFID reader embedded in a hand glove (See Fig. 7 from [20], reproduced with permission).

As the shopper moves around the grocery store, the RFID reader in the hand glove reads IDs from the products, looks them up in a store database through a Wi-Fi connection, and gives instructions to the user. The primary purpose of the device is store browsing. When the shopper moves his or her hand along a shelf, the PDA will deliver messages such as "passing dairy section," "passing coffee section," etc. When the user is inspecting a product, the user will receive the individual package's price, weight, ingredients, and nutritional data presumably from the RFID tag read from the package.

As an accommodation system, iCARE does not meet DR1 (mobile product selection), insomuch as there appears to be no discussion of this problem in the references. The system partially addresses DR2 (store navigation) and DR3 (product search), because RFIDs on individual products can be used as navigational and search cues. However, since the project's primary focus is on product browsing, it is unclear whether store navigation and product search have been actually implemented or ever evaluated in a real supermarket.

The system meets DR4 (product identification) on the assumption that the RFID reader can always read the product's tag. This assumption may not hold for many liquid 
low-end products with metal cases that refract and reflect RF waves. The system partially meets DR5 (utilization of existing devices) in that most technologies appear to rely on completely-off-the-shelf components, except, of course, the hand glove with an embedded RFID reader.

The system does not meet DR6 (minimal environmental adjustment). A fundamental assumption underlying the system's design is that every single item in the store has an RFID tag, which, as we argue in Section 4, presents a significant environmental adjustment cost to modern supermarkets in the short term and an equally significant social acceptance cost in the long term. Furthermore, to support timely delivery of information on prices, weights, ingredients, nutritional data, and store sections, iCARE will need access to the store's inventory control system, which some supermarkets may not be willing to provide.

\subsection{Trinetra}

Trinetra is a project at Carnegie Mellon University [21, 22] whose long-term objective is to develop a mobile system that enables people with visual impairments to shop independently. The system is intended to handle both barcodes and RFID tags, if and when the latter will be available in supermarkets. The system's hardware consists of a Nokia mobile phone, a Bluetooth headset, a Baracoda IDBlue Pen, a Baracoda Pencil, and a Windows-based server. The Baracoda Pencil is used to scan barcodes, and the Baracoda IDBlue Pen is used to scan RFID tags.

The primary use case of the system starts with the shopper scanning a UPC barcode with a Baracoda Pencil and retrieving a description of the product. The data are passed wirelessly to and from the mobile phone via Bluetooth. The system on the phone first checks a local cache to see if the barcode has previously been scanned. If the barcode is not in the mobile application's cache, a request is sent to the remote server, which also has a cache of UPC barcodes. If the barcode is in the server's cache, the information is returned to the phone. If the barcode is in neither cache, a public UPC database is contacted by the server to retrieve the necessary information.

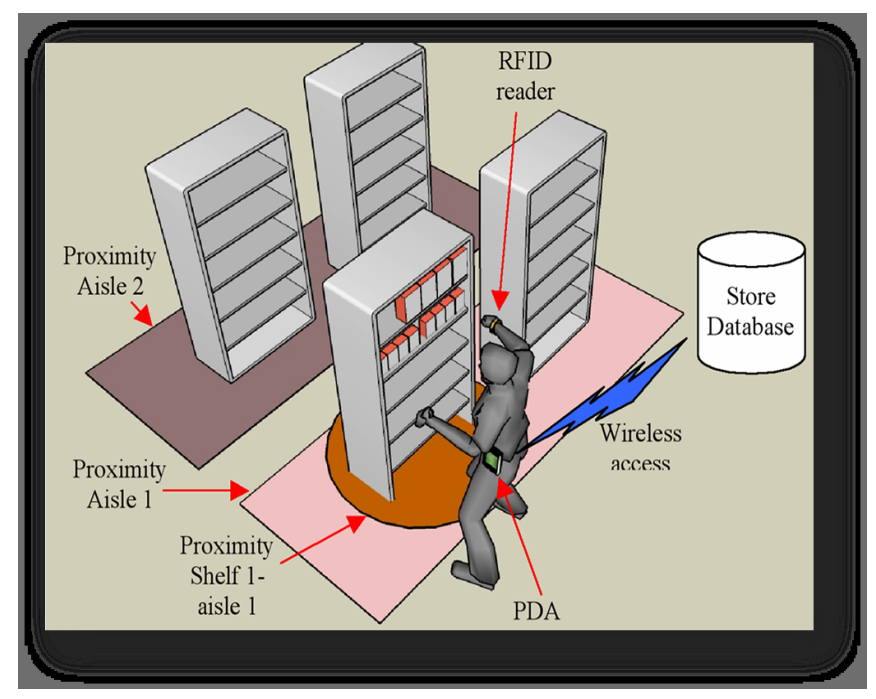

Fig. (7). iCARE framework.
Trinetra partially meets DR1 (mobile product selection) in that the literature offers some evidence that the shopper can select products from the phone. It is unclear, however, if the selection is made from the prepared list or from a database of products. The system does not meet DR2 (store navigation): the shopper is assumed to be able to navigate to the product's location independently or ask for assistance in the store. This assumption may be true in a small convenience store but it is unlikely to hold in a modern supermarket.

The system does not meet DR3 (product search) in that there does not seem to be any explicit guidance provided by the system to the shopper at run time. The system partially meets DR4 (product identification): the shopper can identify the found product by locating and scanning its barcode with the Baracoda pencil. Since the only reported informal singlesubject study was done at a small campus store with two aisles, more empirical evidence is needed to discover how successfully VI shoppers can use the Baracoda pencil to identify products. Our own laboratory studies with the BaraCoda pencil indicate that it may be difficult for both blind and blindfolded individuals to scan shelf and product barcodes with it [23].

The system meets DR5 (utilization of existing devices). To the best of our knowledge, the Trinetra team was the first to advocate the mobile phone platform for accessible blind shopping.

Whether Trinetra meets DR6 depends on which version of the system will be ultimately adopted. While the barcode version of the system meets DR6 (minimal environmental adjustment), provided that the supermarket agrees to install and maintain the UPC barcode server, the RFID version does not, because item-level RFID tagging will require a major environmental adjustment on the part of supermarkets.

\subsection{IBM's Patent}

In 2002, IBM acquired a patent on a method to provide VI shoppers with location and item identification at sites that have barcode labels [24]. The patent describes a portable unit that assists VI shoppers at stores by providing them, through speech synthesis, with information on their locations from various barcode labels. The location of each barcode label is stored in a database on the portable unit.

The portable unit is claimed to be capable of determining a path between the shopper's current location and the location of the item that the shopper wants to find and of describing the path through speech synthesis. The shopper can select items from a shopping list on the portable unit.

To become operational, the system appears to need barcode directories at both ends of each aisle similar to building directories. Each barcode directory will list all barcode labels in a specific aisle. It is not clear from the text of the patent which barcode labels will be in these directories - UPC or MSI or both.

Since, as far as we know, IBM has never built an actual prototype of this device and tested it in a real supermarket, it is difficult to tell how well a system built according to the specifications outlined in the patent will meet the design requirements. The patent does not seem to meet DR1 
(mobile product selection) as it mentions only the shopper selecting items from a target shopping list that already resides on the device. DR2 (store navigation) appears to be met in that the device is claimed to generate verbal route directions to various locations in the store. Presumably, the shopper will have to scan some barcode label in the store to inform the device of his or her present location and then select a product from the stored shopping list. The device will retrieve the location of the target product from its database, plan a path from the shopper's location to the product's location, and verbalize it to the shopper.

While the patent does not appear to address DR3 (product search), one can easily conjecture various means of meeting this requirement given the rich information in the device's barcode database. Thus, it is reasonable to assume that DR3 is, at the very least, partially met.

It is unknown whether the device built according to the patent's specifications will meet DR4 (product identification). It will ultimately depend on the actual means of obtaining barcodes from individual packages. Nor can it be known at this point whether the actual realization of the concept will meet DR5 (utilization of existing devices).

The conceptual design outlined in the patent does not meet DR6 (minimal environmental adjustment). The assumption that both ends of every aisle have detailed barcode directories incurs a high maintenance cost. Many products regularly change their locations; some products are discontinued and replaced with others. It is difficult to see how these changes will find timely reflection in the barcode directories. Furthermore, the patent does not take into account the fact that many supermarkets use the physical space at the end of each aisle for stacking products or for placing advertisement boards.

\subsection{Comparison Summary}

Table 3 summarizes how RoboCart, ShopTalk, GroZi, iCARE, Trinetra, and IBM's device and meet the design requirements summarized in Table 2 . The plus sign means that a given system meets a given design requirement; the minus sign means that a given system does not meet a given design requirement; the plus/minus means that a given system partially meets a given design requirement; the question mark means that it is unknown whether the system meets a specific requirement. We also added a column on field evaluations to reflect whether a specific system was tested in a real store with at least ten visually VI participants.

\section{WHAT ABOUT RFID?}

Our analysis of accessible shopping solutions for blind and VI individuals would be incomplete with no mention of Radio-Frequency Identification (RFID). The topic of RFID is quite polarizing. The technology has many vocal proponents and equally vocal opponents who frequently speak past each other and are unwilling to address each other's concerns and critiques.

RFID has been proposed as a technology of choice for the Electronic Product Code (EPC), a global network of objects under a universal labeling system [25]. Two competing organizations work on international RFID standards: EPCGlobal and the ISO. An RFID system consists of a set of tags and an antenna connected to a reader which, in turn, is connected to a data processor. Tags have chips that respond to radio waves transmitted by readers. Tags can be passive (no external power supply) or active (external power supply). Tags have unique IDs which identify tagged items. Since practical RFID use in accessible blind shopping requires passive item-level tagging, i.e. every single product in a supermarket must have a passive tag, we will focus on the ultra high frequency (UHF) tags, the class that includes passive item-level tags.

Wal-Mart was the first major supermarket chain to champion item-level RFID. In 2005, it mandated its top 100 suppliers to start using item-level RFID [26]. The date was looked upon as a watershed event in the industry. Yet, it arrived and quietly passed. To revive RFID adoption, Sam's Club required some of its suppliers on January 7, 2008, to apply EPC RFID tags to distribution center shipments [27]. The letter outlined fines ranging from $\$ 2$ to $\$ 3$ for each nontagged pallet. This was the first time Wal-Mart, which owns Sam's Club, announced monetary penalties for suppliers for not following RFID tagging guidelines. The suppliers did not comply. In a letter dated January 15, 2009 [28], Sam's Club made case-level tagging optional and the requirement for item-level tagging has been postponed indefinitely. The fine was slashed to $\$ 0.12$ per pallet, which could be construed as a tacit admission of the fact that RFID tagging was not a viable option even in the presence of steep mandatory fines.

The reasons for slow acceptance of RFID by many suppliers are multiple and varied. In this article, we will address only a few. Interested readers are referred to excellent analyses offered in [29]. Many countries have

Table 3. Comparison of RoboCart, Trinetra, ShopTalk, iCARE, and GroZi

\begin{tabular}{|c|c|c|c|c|c|c|c|}
\hline & DR1 & DR2 & DR3 & DR4 & DR5 & DR6 & Field Evals \\
\hline \hline RoboCart & $+/-$ & + & $+/-$ & $+/-$ & - & - & + \\
\hline ShopTalk & - & + & + & $+/-$ & - & $+/-$ & + \\
\hline GroZi & - & $+/-$ & $+/-$ & $+/-$ & - & - \\
\hline iCARE & - & $+/-$ & $+/-$ & + & $+/-$ & - & + \\
\hline Trinetra Barcode & $+/-$ & - & - & $+/-$ & + & + & - \\
\hline Trinetra RFID & $+/-$ & - & - & + & + & - & - \\
\hline IBM & - & + & $+/-$ & $?$ & $?$ & - \\
\hline
\end{tabular}


auctioned large portions of the UHF spectrum to cellular phone service providers for high license fees. For many suppliers, buying that spectrum back for RFID use is impractical, if not impossible. It does not make much business sense to invest in RFID use in global supply chains when tags in source countries cannot be read in target countries due to incompatible UHF spectrum bands.

Continuing lack of convincing business cases that satisfy all parties, not just retailers and RFID manufacturers, is another major reason why many suppliers steer clear of RFID. The manufacturing costs for tags and readers remain prohibitive for tagging all but high-value products unless the tagging is subsidized by the retailer. According to many independent RFID analysts, significant cost reduction will be slow due to numerous unresolved intellectual property issues and manufacturing costs [30].

The future of item-level RFID tagging remains, at best, uncertain due to technical problems. Liquid low-end products, such as shampoos, juices, sodas, and canned goods with metal cases, refract and reflect RF waves. If UHF radio waves propagate toward liquid, they refract into it; if they pass onto metal, they are reflected. Several research solutions have been proposed; none have been shown to work consistently [31].

Product packaging with RFID tags poses a clear substantial threat to the recycling of residential and industrial waste: it contaminates host materials and prohibits effective processing of paper, plastic, glass, pallets, boxes, and other supply chain containers. Yet, no recycling provisions for RFID exist and none are planned.

Consumer privacy is another hurdle that the RFID technology will have to overcome. RFID store trials by Benetton, Prada, and Gap were cancelled due to consumer privacy outcry; the same fate awaited Gillette which, in cooperation with Wal-Mart, attempted to place RFID tags on individual razors [32]. A comprehensive study on consumer perception of RFID was conducted by Günther and Spiekermann with two groups of 129 well educated individuals in an RFID-enabled retail environment [33]. The study showed that, regardless of the Privacy Enhancement Technology (PET), consumers felt helpless toward the RFID environment. RFID-related privacy concerns recently resulted in the formation of Consumers Against Supermarket Privacy Invasion and Numbering (CASPIAN), an active non-profit consumer advocacy group that referred to RFID as "paving the way for a totalitarian state" [34]. These sentiments clearly indicate that item-level RFID tagging has a high social cost.

Despite numerous research proposals and marketing initiatives, the literature appears to have reached a consensus that technical problems, environmental hazards, and consumer perceptions of trust, privacy, and risk, mixed with fear (the recent vulnerability issues with RFID-enabled U.S. passports furnish yet another good example [35]), remain significant acceptance barriers to RFID item-level tagging. RFID use in retail will likely be limited to tracking containers, not individual items, in the short term.

In the long term, RFID should by no means be discounted as it has many vocal proponents in $R \& D$ and retail. For example, the RFID outlook may gradually change as Wal-Mart begins to use RFID tags to track individual pairs of jeans and underwear, which some experts view as the first step in implementing a comprehensive RFID-based inventory control [36]. If this trial succeeds, it may create the tipping point for RFID tags' prices and force other retailers to consider RFID tags in their inventory control systems.

\section{WHERE TO NOW?}

Several commercial products already exist on the market and can be productively used by blind and VI people in supermarkets and other stores. Looky [37] and OPAL [38] are electronic magnifiers for low-vision people that offer advantages over a regular hand-held magnifying glass, such as zoom and image enhancement. OMNI [39], a commercial product from En-Vision America, is a standalone, talking barcode scanner. It has an on-board database of UPC barcodes and includes extra data such as ingredients and nutrition information for some products. The database updates require an annual paid subscription.

Our analysis of the existing systems shows that no solution meets all the design requirements wanted by VI shoppers. None of the solutions meets DR1 (mobile product selection). While some progress has been recently made [40], more work must be done to meet this design requirement.

The existing solutions appear to display better awareness of the requirements DR2 - DR4 (store navigation, product search, and product identification). This does not appear to be the case with DR5 (utilization of existing devices) yet. This requirement is significant as VI and blind consumers show reluctance to purchase hardware items in addition to those that they already own and know how to operate. As more and more VI individuals become mobile phone users, the accessible shopping R\&D community should take a serious look at adopting the mobile phone as the central computational unit of the proposed solutions.

Definite progress has been made on vision-based barcode recognition algorithms [41]. As the mobile phones become more computationally powerful, these algorithms can successfully be ported to various mobile platforms. What makes vision-based barcode recognition particularly difficult for VI individuals is the problem of camera alignment. More research is needed on vision techniques that recognize partial barcodes in images and give the VI users audio or haptic feedback to align the phone cameras with barcodes [42].

Most algorithms focus on recognizing UPC barcodes. Surprisingly little research has been done on recognizing MSI and other non-UPC barcodes, which is unfortunate, because, as we argued above, such barcodes can be key to independent store navigation and exploration. The small amount of research can be explained by lack of uniform inventory control standards. These barcodes vary from store to store or from store chain to store chain. However, it not unreasonable to conjecture that algorithms can be designed that learn barcode decoders for specific stores from small samples.

The minimal environmental adjustment requirement (DR6) is hard to meet for any accessible shopping system. Any system that purports to give VI and blind shoppers adequate store navigation and product search support is 
likely to be coupled to the store's inventory control system. Since such collaboration may not be forthcoming from some stores or chains, it may be worth it for the accessible shopping R\&D community to think of solutions that do not depend on store collaboration. One such solution may be collaborative knowledge engineering where a group of blind, VI and sighted individuals, over a period of time, create and maintain a database of a specific store in the same way as sighted individuals annotate various regions of the world on sites such as Wikimapia (http://wikimapia.org/).

As our analysis indicates, the existing solutions have very few comprehensive field evaluations. This lack of evaluations makes it more difficult to predict which approaches to accessible blind shopping may translate into real systems. It may be impractical, if not impossible, to run controlled studies in real supermarkets due to the ever fluctuating shopper traffic patterns and indigenous business processes that cannot be interrupted. But, rigorous controlled studies may not be needed. In our opinion, diary studies are particularly well suited for evaluating accessible shopping systems in the target environments and should be pursued.

In conclusion, we would like to offer a few thoughts on the relationship between our analysis and Universal Design (UD). UD is a term created by Ron Mace, architect, who founded and directed the Center for Universal Design at the College of Design at NC State University (http://www.ncsu. edu/dso/general/universal-design.html). The Center has worked on accessible design for environment and products since 1989. UD has seven principles: 1 ) equitable use (utility and marketability to people with diverse abilities); 2) flexibility in use (accommodation of multiple preferences and abilities); 3) simple and intuitive use (easy comprehension regardless of the end user's experience, knowledge, language, or current concentration level); 4) perceptible information (effective communication of necessary information regardless of ambient conditions); 5) tolerance for error (minimization of hazards and adverse consequences of accidental and unintended actions); 6) low physical effort (efficient and comfortable usage with minimum fatigue); 7) size and space for approach and use (provision of sufficient size and space for approach, reach, manipulation, and use regardless of user's body size, posture, and mobility).

How would an accessible shopping system that meets the design requirements proposed in our article (DR1 - D6) fare with respect to the seven UD principles? Such a system would probably meet the first UI principle in that it could be useful not only to the blind and visually impaired but also to the elderly. The second UD principle may also be met insomuch as the system could be used for both spontaneous and planned shopping. It is difficult to tell whether the third principle would be met. For example, if the system is deployed on a smartphone with a touch screen, a significant amount of the blind user's experience will be determined by the touch gesture user interfaces. The system that satisfies the design requirements of store navigation, product search, and identification will, by definition, meet the fourth principle with respect to accessible shopping. In our opinion, all systems analyzed in this article meet the fifth UD principle in that they do not increase the risks and hazards typically associated with blind navigation in dynamic and complex structured indoor environments. The satisfaction of the sixth UD principle of low physical effort is an empirical question that will likely vary from system to system. Finally, the seventh UD principle will be easily met because barcode scanners, smartphones, and gloves are used by people with various hand and body sizes, postures, and mobilities.

\section{SUPPLEMENTAL ONLINE MATERIALS}

Interested readers can examine several online resources related to the systems described in this article. Research videos of blind and VI individuals testing RoboCart and ShopTalk at Lee's Market Place in Logan, Utah are available on the USU CSATL's YouTube channel at http://www.yout ube.com/user/CsatlUSU. The site http:/grozi.calit2.net/ offers a wealth of materials about GroZi. Trinetra's home page at http://www.ece.cmu.edu/ trinetra/ contains several articles and videos. While iCARE does not appear to have its own web site, interested readers are advised to check the site of the Center for Cognitive and Ubiquitous Computing of Arizona State University at http://cubic.asu.edu. Readers interested in UD can take a look at the home page of the Center for Universal Design at the College of Design of NC State University at http://www.ncsu.edu/www/ncsu/design/ sod5/cud/.

\section{ACKNOWLEDGEMENTS}

We would like to thank the anonymous reviewers of this article for their constructive comments. We would like to thank the NFB Chapter in Logan, Utah and especially Sachin Pavithran for helping us organize the two focus groups on accessible blind shopping. We are grateful to Serge Belongie for giving us the pictures of the MoZi box and the GroZi glove and to Vineeth Balasubramanian for the picture of the iCare framework. We would like to thank John Nicholson for reading several drafts of this manuscripts and providing valuable comments. Our research on accessible shopping and blind navigation system has been supported, in part, through the NSF grant IIS-0346880, the NEI/NIH grant R41 EY017516-01A1, and the Community University Research Initiative (CURI) in the State of Utah.

\section{REFERENCES}

[1] Food marketing institute research. The food retailing industry speaks 2006. Food Marketing Institute 2006.

[2] Nicholson J, Kulyukin V, Coster D. ShopTalk: Independent blind shopping through verbal route directions and barcode scans. Open Rehab J 2009; 2: 11-23.

[3] McQuistion L. Rehabilitation Engineering: Ergonomics for one. Ergon Des 1993; 9-10.

[4] Kulyukin V, Gharpure C. Ergonomics for one. In: A robotic shopping cart for the blind proceedings of the acm conference on human robot interaction (HRI). Salt Lake City 2006; pp. 142-9.

[5] Kulyukin V, Gharpure C, Pentico C. Robots as interfaces to haptic and locomotor spaces. Proceedings of the acm conference on human-robot interaction (HRI). Washington DC 2007; pp. 325-31.

[6] Gharpure C. Kulyukin V. Robot-Assisted Shopping for the Blind. Issues in spatial cognition and product selection. Int $\mathrm{J}$ Serv Robotics 2008; 1(3): 237-51.

[7] LaPlante M, Carson D. Disability in the United States: Prevalence and causes. U.S. Department of education, Washington DC, 2000.

[8] Raman TV. Auditory user interfaces. Kluwer Academic Publishers, Boston, Hard Bound 1997; p. 168.

[9] Smith A, Cook J, Francioni J, Hossain A, Anwar M, Rahman M. Nonvisual tool for navigating hierarchical structures. In: 
Proceedings of the ACM SIGACCESS. Accessibility and Computing Conference. Atlanta 2004; 133-9.

[10] Kientz J, Patel S, Tyebkhan A, Gane B, Wiley J, Abowd G. Where's My Stuff? Design and evaluation of a mobile system for locating lost items for the visually impaired. Proceedings of the 8th ACM Conference on Computers and Accessiblity (ASSETS 2006), Portland, Oregon, USA, October 22-25, 2006.

[11] Bronfenbrenner U, Kazdin AE, Eds. Ecological systems theory. Encyclopedia of Psychology. Vol 3. Washington, DC, US: American Psychological Association, Oxford University Press 2000; pp. 129-33.

[12] Kulyukin V, Gharpure C, Nicholson J. RoboCart: Toward robotassisted navigation of grocery stores by the visually impaired. In: Proceedings of the IEEE/RSJ International Conference on Intelligent Robots and Systems (IROS). Edmonton, Canada: IEEE Press 2005; pp. 2845-50.

[13] Kulyukin V, Gharpure C, Nicholson J, Osborne G. Robot-Assisted way-finding for the visually impaired in structured indoor environments. Autonomous Robots 2006; 21(1): pp. 29-41.

[14] Kulyukin V, Gharpure C, Pentico C. Robots as interfaces to haptic and locomotor spaces. Proceedings of the ACM/IEEE international conference on Human-robot interaction (HRI). Washington, DC, USA: ACM 2007; pp. 325-31.

[15] Freundschuh S, Egenhofer M. Human conceptions of spaces: Implications for Geographic Information Systems. Trans GIS 1997; 2(4): 361-75.

[16] Nicholson J, Kulyukin V. ShopTalk: Independent blind shopping = verbal route directions + barcode scans. Proceedings of the $30^{\text {th }}$ Annual Conference of the Rehabilitation Engineering and Assistive Technology Society of North America (RESNA). Phoenix, Arizona 2007.

[17] Merler M, Galleguillos C, Belongie S. Recognizing groceries in situ using in vitro training data. SLAM, Minneapolis, MN 2007.

[18] Winlock T, Christiansen E, Belongie S. Toward real-time grocery detection for the visually impaired, Proceedings of the Computer Vision Applications for the Visually Impaired Workshop (CVAVI), San Francisco 2010

[19] Krishna S, Panchanathan S, Hedgpeth T, Juillard C, Balasubramanian V, Krishnan NC. A wearable wireless rfid system for accessible shopping environments. $3^{\text {rd }}$ Intl Conference on BodyNets'08; Tempe, AZ 2008.

[20] Krishna S, Balasubramanian V, Krishnan NC, Hedgpeth T. The iCARE Ambient interactive shopping environment, California State University, Northridge, Center on Disabilities' 23rd Annual International Technology and Persons with Disabilities Conference (CSUN). Los Angeles, CA 2008.

[21] Lanigan PE, Paulos AM, Williams AW, Rossi D, Narasimhan P. Trinetra: Assistive technologies for grocery shopping for the blind. In: International IEEE-BAIS Symposium on Research on Assistive Technologies (RAT). Dayton, OH 2007.

[22] Narasimhan P. Assistive Embedded technologies. IEEE Computer 2006; vol. 39: pp. 85-87.
[23] Janaswami K. ShopMobile: A mobile shopping aid for visually impaired individuals, M.S. Report, Department of Computer Science. Utah State University, Logan, Utah 2010.

[24] Conzola VC, Cox AR, Ortega KA, Sluchak TJ. Providing location and item identification data to visually impaired shoppers in a site having barcode labels. U.S. Patent US 20020158133A1. Oct. 31, 2002.

[25] Finkenzeller K. RFID Handbook-Fundamentals and applications in contactless smart cards and identification. Wiley, New York 2003.

[26] Wu NC, Nystrom MA, Lin TR, Yu HC. Challenges to Global RFID Adoption. Technovation 2006; 26: 1317-23.

[27] Roussos G, Kostakos. RFID in pervasive computing: State-of-theart and outlook. Pervasive Mobile Computing 2009; 5: 110-31.

[28] Burnell J. Sam's club letter outlines changes to RFID requirements. RFID Update, 2009. Available for download at http://www.rfidup date.com/articles/index.php?id=1734.

[29] Roussos G. Enabling RFID in retail. Computer 2006; vol. 39: pp. 25-30.

[30] Stapleton-Gray R. Scanning the horizon: A skeptical view of RFIDs on the shelves. 2005; Available from: www.rfidprivacy.us/ 2003/papers/stapleton-gray3.pdf.

[31] Renegar B, Michael K. The RFID value proposition. Proceedings Of Collecter Iberoamérica Madrid, Spain 2008.

[32] Langheinrich M. RFID and privacy. In: Security, Privacy and Trust In Modern Data Management. Petkovic M, Jonker W, Eds. Berlin: Springer-Verlag 2006.

[33] Günther O, Spiekermann S. RFID and the Perception of Control: The consumer's view. Comm ACM 2005; 48 (9): 73-6.

[34] CASPIAN - Consumers against supermarket privacy invasion and numbering, www.nocards.org, www.spychips.com.

[35] Ramos A, Scott W, Scott W, Lloyd D, O’Leary K, Waldo J. A Threat analysis of RFID passports. Comm ACM 2009; 52(12): 3842.

[36] Bustillo M. Wal-Mart radio tags to track clothing. Wall Street J 2010;26.

[37] Looky handheld magnifier, http://www.steller-technology.co.uk/ looky.php.

[38] OPAL Ultra-portable video magnifier, http://www.freedomscienti fic.com/products/lv/opal-productpage.asp.

[39] i.d. mate OMNI, http://www.envisionamerica.com/idmate/.

[40] Kulyukin V, Gharpure C. User intent communication in robotassisted shopping for the blind. In: Advances in Human-Robot Interaction. Kulyukin V. Ed. IN TECH, 2009; pp. 325-42.

[41] Tekin E, Coughlan JM. An algorithm enabling blind users sto find and read barcodes. In: Proceedings of the IEEE Workshop on Applied Computer Vision 2009; pp. 1-8.

[42] Kulyukin V, Kutiyanawala A. Eyes-free barcode localization and decoding for visually impaired mobile phone users. Proceedings of the International Conference on Image Processing, Computer Vision, \& Pattern Recognition (IPCV). Las Vegas, NV: CSREA Press 2010; 1: pp. 130-5,

(C) Kulyukin and Kutiyanawala; Licensee Bentham Open.

This is an open access article licensed under the terms of the Creative Commons Attribution Non-Commercial License (http: //creativecommons.org/licenses/bync/3.0/) which permits unrestricted, non-commercial use, distribution and reproduction in any medium, provided the work is properly cited. 